\title{
ANSIEDAD Y DEPRESIÓN EN NIÑOS DIAGNOSTICADOS CON CÁNCER
}

\author{
PAMELA CABRERA, BEATRIZ URRUTIA, VERÓNICA VERA, \\ MÓNICA ALVARADO y PABLO VERA-VILLARROEL
}

\author{
Escuela de Psicología \\ Universidad de Santiago de Chile, USACH
}

(Aceptado en noviembre de 2004)

\begin{abstract}
El cáncer es una de las principales causas de muerte infantil. Esta enfermedad constituye una situación grave y estresante provocando en el niño estados emocionales que generan malestar psicológico tales como ansiedad y depresión. El objetivo de este trabajo fue evaluar los síntomas depresivos y ansiosos en niños con cáncer entre los 6 y 12 años controlando las variables de sexo, edad y tiempo transcurrido desde el diagnostico. Para ello se aplicó el cuestionario de depresión infantil (CDI) de Kovacs y el inventario de ansiedad estado-rasgo infantil (STAIC) de Spielberger. La muestra estuvo compuesta por 65 niños ( 36 de sexo masculino y 29 de sexo femenino). Se analizó la relaciones y diferencias considerando sexo, edad y tiempo desde que fueron diagnosticados con la enfermedad. No se encontraron diferencias en ansiedad rasgo y depresión. Se encontraron diferencias en ansiedad estado y el tiempo transcurrido desde el momento de ser diagnosticado.
\end{abstract}

Palabras clave: Cáncer, niños, ansiedad, depresión

\section{Anxiety and depression in children with a diagnosis of cancer}

Cancer is one of the main causes of death in children. This illness constitutes a crisis situation that is very stressful, causing the child to experience emotional states that lead to psychological distress such as anxiety and depression. The goal of this work was to assess anxious and depressive symptoms in children between 6 and 12 years old who suffer from cancer, controlling for sex, age and time elapsed since the child had been diagnosed. We used Kovac's child depression inventory (CDI) and Spielberger's trait-anxiety inventory for children (STAIC). The sample included 65 children (36 males and 29 females). Differences and associations of the variables considering sex, age, and time elapsed since the diagnosis were analyzed. The results showed no significant differences in trait anxiety and depression. But there were significant differences in trait-anxiety as a function of time elapsed since the diagnosis.

Key words: Cancer, children, anxiety, depression.

\section{INTRODUCCION}

A partir de la década del 60 las investigaciones realizadas en oncología permitieron comenzar tratamientos más efectivos en las neoplasias pediátricas (leucemia y tumores sólidos). Paralelamente a estos avances en distintos países de Latinoamérica se crean unidades hospitalarias destinadas especialmente al diagnóstico y tra-

Correspondencia: Pablo E. Vera-Villarroel, Escuela de Psicología, Universidad de Santiago de Chile USACH, Avenida Ecuador $3650,3^{\text {er }}$ Piso, Santiago, Chile. Correo-e: pvera@lauca.usach.cl tamiento del cáncer infantil. En Chile alrededor de 1968 los hospitales Luis Calvo Mackena y Roberto del Rio inician el funcionamiento de sus unidades oncológicas.

Desde ese momento, han existido importantes desarrollos en este ámbito, generándose las condiciones para que en toda Latinoamérica se realice diagnostico y tratamiento a los niños afectados por esta enfermedad, dependiendo de las características de cada país. Sin embargo, en Latinoamérica el cáncer se ha adjudicado un lugar significativo en la mortalidad infantil llegando a ser una de las 
principales causas de muerte infantil. Según la Sociedad Latinoamericana de Oncología Pediátrica (SLAOP), las cifras de las frecuencias de las neoplasias en Latinoamérica son relativas debido a que no se tienen registros estadísticos rigurosos en algunos países, por lo que los datos existentes son aproximados.

Del total de cánceres que se dan en la población mundial, el 3 a $4 \%$ corresponde a neoplasias pediátricas; sin embargo, de todas las muertes ocurridas a causa de esta enfermedad sólo el $5 \%$ afecta a la población infantil, por lo que se evidencia una mejor supervivencia que los adultos. Por otra parte, en términos de distribución de acuerdo al género, a nivel mundial, la incidencia de cáncer infantil es mayor en hombres que en mujeres (Wagner y Antic, 1997; Pratt, 1985).

En cuanto a las características de esta enfermedad, el cáncer infantil se caracteriza por ser altamente invasivo, tener un rápido crecimiento y desarrollar metástasis de manera temprana. Es por esto que la invasión de tejidos sanos es extremadamente rápida, lo que deriva en una sintomatología sistémica (por ejemplo, decaimiento, pérdida de peso, etc.). Así también los procesos neoplásicos pediátricos también se caracterizan por poseer una alta sensibilidad a la quimioterapia y radioterapia, y por lo tanto, existirían buenas posibilidades de un tratamiento curativo o paliativo de realizarse oportunamente.

El tratamiento actual del cáncer se basa en un enfoque de la enfermedad en dos niveles, uno local y otro sistémico. La enfermedad local se trata con cirugía y radioterapia, y la sistémica (metástasis) con quimioterapia. Sólo con este enfoque multisistémico combinando una serie de terapias, se ha logrado disminuir la mortalidad de cáncer pediátrico. Esto ha significado lograr, tanto la disminución de importantes secuelas y complicaciones, como también, bajar las dosis de cada uno de los tratamientos involucrados (Marina, 1997; Berg, Grisell, DeLaney y Balis, 1991).

Por otra parte, el sufrir una enfermedad ha sido considerada como una situación estresante. Esto aumenta cuando se trata de enfermedades crónicas como el cáncer, puesto que esta enfermedad amenaza directamente la supervivencia de la persona. En este sentido, a menudo el curso y pronóstico de la enfermedad son inciertos, su padecimiento puede suponer mutilaciones para el enfermo, los tratamientos interrumpen la vida cotidiana produciendo cambios importantes en los hábitos habituales de los pacientes y sus familias (Latorre y Beneit, 1994; Galán, Pérez San Gregorio y Blanco, 2000; Pérez, Martín, Gallego y Santamaría, 2000; Vera-Villarroel, Alvarado, Cabrera, Urrutia y Vera, 2000; Baider, 2003; Barra, 2003; Martín, Sánchez y Sierra, 2003). Específicamente en el caso de los niños la experiencia de sufrir cáncer y estar sometido al tratamiento es altamente traumática, más aún cuando el niño no tiene un exacto conocimiento de lo que sucede. El menor debe enfrentarse a situaciones estresantes como los procedimientos médicos invasivos y dolorosos, períodos de hospitalización, interrupción de la asistencia a la escuela e inclusive períodos de aislamiento. Producto de ello, se producen en el niño sentimientos de temor, angustia, ira, culpa y pánico, entre otros. También suelen surgir fantasías relacionadas principalmente con daño corporal, temor a morir o a quedar con algún defecto entre otras (Vilches, Yañez y González, 1996). Sin embargo, las reacciones psicológicas mayormente observadas en pacientes con cáncer son la ansiedad y la depresión. Distintos autores señalan que luego de realizado el diagnóstico de cáncer, es predecible que se produzca una reacción emocional aguda caracterizada por la aparición de síntomas ansiosos y depresivos (Lizasoaín y Polaino-Lorente, 1990; Cruzado y Olivares, 1996; Beneit, 1994; Rona y Vargas, 1994). 
Por otra parte, algunos autores plantean que las fuentes de ansiedad varían dependiendo de la etapa de desarrollo en la que se encuentre el niño. Así los niños menores de 6 años presentarían ansiedad frente a la separación de las figuras significativas, los niños entre 6 y 10 años manifestarían respuestas ansiosas ante los procedimientos médicos y en los niños mayores de 10 años se presentarían reacciones ansiosas frente a la pérdida de funciones corporales y la muerte (Aravena, Riedemann y Vásquez, 1993; Valverde y Vasquez, 1991; Sandin, Chorot, Valiente y Santed, 1998). De la misma forma, las reacciones emocionales se presentarían en los diferentes períodos por los que evoluciona la enfermedad (Cruzado y Olivares, 1996; Rona y Vargas, 1994; Ulloa, 1993).

Considerando esta enfermedad como un estado altamente estresante, que los pacientes y específicamente los niños deben afrontar esta situación, y que las reacciones emocionales pueden variar dependiendo de la fase en la cual se encuentren en relación con la enfermedad, el objetivo del presente estudio fue evaluar los estados emocionales en niños que presentan cáncer en distintos periodos de tiempo. De la misma forma, evaluar si existen diferencias de acuerdo al sexo y edad de los niños.

\section{MÉTODO}

\section{Participantes}

La muestra estuvo compuesta por 65 niños entre seis y doce años de edad ( 29 chicas y 36 chicos) que asistían a los servicios de Oncología de dos hospitales públicos de la ciudad de Santiago de Chile. Todos los niños contaban con el consentimiento de sus padres para participar en el estudio. Media de edad = 8,87 (DT = 2,04).

\section{Instrumentos}

Inventario de Ansiedad-Rasgo para niños STAIC (Spielberguer, 1973,1986). Cuestionario de autoinforme compuesto por dos escalas de 20 preguntas tipo Lickert (tres alternativas de respuesta en cada pregunta). La escala de AnsiedadEstado evalúa los estados transitorios de ansiedad, es decir, sentimientos subjetivos conscientemente percibidos de aprehensión, tensión y preocupación que varían en intensidad y fluctúan en el tiempo. La escala de Ansiedad-Rasgo, evalúa diferencias individuales relativamente estables en la predisposición a la ansiedad. En este estudio se aplicó la adaptación chilena de Cambiaso y Villaseca (1992).

Cuestionario de Depresión Infantil (CDI; Kovacs, 1992; Cáceres y Collado, 1994). Es un inventario de autoinforme para evaluar la depresión infantil de 27 preguntas tipo Lickert, cada una de las cuales consiste en tres afirmaciones entre las que el niño debe elegir aquellas que mejor describa su situación en las últimas dos semanas. Este cuestionario evalúa la presencia y severidad de una amplia gama de síntomas conceptualizados como de importancia clínica en la depresión en contextos que son relevantes para el niño.

Encuesta para padres y/o acompañante. Construida para este estudio con el objetivo de identificar aspectos psicosociales que permitieran caracterizar la muestra.

\section{Procedimiento}

El presente estudio se realizó en tres fases. Durante la primera de ellas se identificó a los pacientes entre seis y ocho años de edad que asistían a los servicios de Oncología de dos hospitales. Posteriormente fueron citados sus padres a los cuales se les presentó el objetivo del estudio y se les solicitó su consentimiento por escrito para la participación de los 
Tabla 1. Tipo de cáncer y frecuencia y porcentajes de la muestra

\begin{tabular}{lcc}
\hline \multicolumn{1}{c}{ Tipo de cáncer } & Frecuencia & Porcentaje \\
\hline Leucemia linfobástica aguda & 16 & 24,6 \\
Leucemia linfobástica & 5 & 7,7 \\
Tumores (Wilms, selar, medular, ovárico) & 6 & 9,2 \\
Leucemia mieloide aguda & 6 & 9,2 \\
Histiocitosis & 4 & 6,2 \\
Osteosarcoma & 4 & 6,2 \\
Linfoma Hogkin & 3 & 4,6 \\
Leucemia mieloblástica & 2 & 3,1 \\
Linfoma no Hogkin & 3 & 4,6 \\
Meduloblastoma & 2 & 3,1 \\
Sarcoma Ewings & 2 & 3,1 \\
Rabdiomiosarcoma & 2 & 3,1 \\
Otros & 10 & 15,4 \\
\hline TOTAL & 65 & 100 \\
\hline
\end{tabular}

menores en la investigación. Una vez obtenida la autorización de los padres se aplicaron los cuestionarios a los niños de modo individual en el momento que asistían a los controles en el hospital y de la misma forma se aplicó el cuestionario a los padres o acompañantes.

Para el análisis de datos se realizó análisis tanto intra como intergrupal. Se realizaron análisis descriptivos a través de medidas de tendencia central, de dispersión y de posición. Se efectuó, además, un análisis comparativo mediante ANCOVA y un análisis correlacional mediante el coeficiente de Pearson. Para llevar a cabo un análisis más especifico se realizó la prueba de Tukey.

\section{RESULTADOS}

Los datos obtenidos en relación al tipo de cáncer y su frecuencia en la muestra se pueden observar en la Tabla 1, destacándose la leucemia linfobástica aguda con mayores casos (16), y los tumores y leucemia mieloide aguda, ambos con seis casos cada una.

En cuanto a la distribución por sexo los niños fueron 36 niños de sexo masculino lo que equivale al $55,4 \%$ y 29 correspondían al sexo femenino $(44,6 \%)$. Respecto a la edad la media fue de 8,87 años con una moda de 9 años. Para controlar el posible efecto de la variable edad se establecieron tres grupos de acuerdo al desarrollo cognitivo, nivel de memoria, pensamiento y lenguaje (Alexander, Roodin y Gorman, 1994). De esta forma se puede observar en la Tabla 2 la conformación de los grupos y los valores en las diferentes variables emocionales evaluadas.

En relación al tiempo desde que los niños fueron diagnosticados, la media fue de 34,8 meses con una desviación típica de 29,87. La moda fue de 33 meses.

Tabla 2. Descripción de los grupos conformados de acuerdo a la edad (frecuencia, porcentaje) y niveles de depresion y ansiedad [medias y desviaciones típicas (entre paréntesis)]

\begin{tabular}{lcccccc}
\hline & Años & Frecuencia & Porcentaje & Depresión & Ansiedad Estado & Ansiedad Rasgo \\
\hline Grupo I & 6 & 12 & $18,5 \%$ & $13,08(8,50)$ & $34,00(5,72)$ & $39,16(10,24)$ \\
GrupoII & $7-9$ & 27 & $41,5 \%$ & $10,22(5,41)$ & $29,33(4,87)$ & $33,77(6,33)$ \\
Grupo III & $10-12$ & 26 & $40 \%$ & $11,84(5,80)$ & $30,42(5,57)$ & $36,53(5,36)$ \\
\hline TOTAL & & 65 & $100 \%$ & $11,40(6,21)$ & $30,63(5,50)$ & $35,87(7,04)$ \\
\hline
\end{tabular}


Tabla 3. Descripción de los grupos por tiempo desde el momento de recibir el diagnóstico de la enfermedad (percentil, frecuencia y porcentajes) y niveles de depresión y ansiedad [medias y desviaciones típicas (entre paréntesis)]

\begin{tabular}{ccccccc}
\hline $\begin{array}{c}\text { Tiempo del } \\
\text { diagnóstico } \\
\text { (meses) }\end{array}$ & Percentil & Frecuencia & Porcentaje & Depresión & $\begin{array}{c}\text { Ansiedad } \\
\text { Estado }\end{array}$ & $\begin{array}{c}\text { Ansiedad } \\
\text { Rasgo }\end{array}$ \\
\hline $\mathbf{0 , 5 - 5}$ & 10 & 6 & 9,2 & $10,00(6,22)$ & $29,83(5,49)$ & $33,66(7,71)$ \\
$6-10$ & 20 & 6 & 9,2 & $12,00(9,65)$ & $31,16(4,62)$ & $33,33(3,44)$ \\
$11-12$ & 30 & 5 & 7,7 & $10,60(4,15)$ & $34,00(2,54)$ & $36,00(8,45)$ \\
$13-19$ & 40 & 9 & 13,8 & $11,00(5,93)$ & $31,55(5,89)$ & $35,00(5,04)$ \\
$20-25$ & 50 & 6 & 9,2 & $16,33(6,88)$ & $34,00(5,93)$ & $43,83(9,26)$ \\
$26-32$ & 60 & 7 & 10,8 & $10,28(3,77)$ & $30,28(5,61)$ & $34,42(6,85)$ \\
$33-34$ & 70 & 6 & 9,2 & $14,16(8,3)$ & $33,66(6,34)$ & $33,83(8,03)$ \\
$35-62$ & 80 & 7 & 10,8 & $10,85(5,66)$ & $30,85(3,62)$ & $38,57(6,72)$ \\
$63-80$ & 90 & 6 & 9,2 & $6,83(4,57)$ & $22,83(3,48)$ & $32,33(7,99)$ \\
& 100 & 7 & 10,8 & $12,14(4,98)$ & $28,57(3,64)$ & $37,71(3,25)$ \\
\hline TOTAL & & & & $11,4(6,21)$ & $30,63(5,5)$ & $35,87(7,04)$ \\
\hline
\end{tabular}

El tiempo mínimo de diagnóstico observado en la muestra fue de 0,5 meses y el tiempo máximo transcurrido desde el diagnóstico fue de 117 meses. Para controlar la variable tiempo de la enfermedad se conformaron diez grupos considerando como criterio de agrupación la distribución por percentil. Los grupos conformados se pueden observar en la Tabla 3. Las medias y desviaciones típicas de las variables emocionales también se pueden observar en dicha tabla.

Con relación a las variables psicológicas se analizaron de acuerdo al sexo, edad, grupos de edad y tiempo del diagnóstico. En este sentido se puede observar en la
Tabla 4 los índices en ansiedad estado, rasgo y depresión de acuerdo al sexo.

Para examinar la relación entre las variables se realizó un análisis de correlación de Pearson, el cual mostró una relación significativa entre las variables emocionales. Sin embargo, no se encontró una relación estadísticamente significativa entre edad y las variables depresión, ansiedad estado y ansiedad rasgo. Sí se encontró una relación negativa estadísticamente significativa entre tiempo de diagnóstico y ansiedad estado. No había una relación significativa entre el tiempo de diagnóstico con depresión y ansiedad rasgo. Véase la Tabla 5.

Tabla 4. Descripción de las medias y desviaciones típicas (entre paréntesis) en las variables emocionales de acuerdo al sexo

\begin{tabular}{llll}
\hline Variables emocionales & Niñas $N=29$ & Niños $N=36$ & Total $N=65$ \\
\hline Depresión & $10,72(6,07)$ & $11,94(6,36)$ & $11,40(6,21)$ \\
Ansiedad Estado & $30,37(5,57)$ & $30,38(5,51)$ & $30,63(5,50)$ \\
Ansiedad Rasgo & $36,03(7,71)$ & $35,75(6,57)$ & $35,87(7,04)$ \\
\hline
\end{tabular}

Tabla 5. Correlaciones entre las variables emocionales, edad y tiempo del diagnóstico.

\begin{tabular}{lcccc}
\hline & Depresión & Ansiedad Estado & Ansiedad Rasgo & Edad \\
\hline Ansiedad Estado & $0,63^{\star *}$ & & & \\
Ansiedad Rasgo & $0,38^{\star *}$ & $0,29^{\star}$ & & \\
Edad & $-0,29$ & $-0,18$ & $-0,07$ & \\
Tiempo del diagnóstico & $-0,03$ & $-0,25^{\star}$ & 0,08 & 0,13 \\
\hline
\end{tabular}

* $\mathrm{p}<0,05 * \star \mathrm{p}<0,01$ 
En el análisis de covarianza (ANCOVA) se consideraron las variables independientes sexo y tiempo de diagnóstico, controlándose el efecto de la variable edad codificada por grupo sobre las variables ansiedad estado-rasgo y depresión. Para llevar a cabo un análisis más específico de comparación entre los grupos se aplicó la prueba post-hoc de Tukey.

En relación con la variable ansiedad estado la ANCOVA indicó que no se encontraron efectos de interacción entre sexo y tiempo de diagnóstico. Tampoco se encontraron diferencias por sexo. Sin embargo se encontraron diferencias con respecto al tiempo de diagnóstico $(\mathrm{F}=$ $2,21, \mathrm{p}<0,03)$. El análisis de Tukey llevado a cabo para identificar las diferencias en los niveles de ansiedad estado entre los diferentes meses mostró que los niños ubicados entre los 63-80 meses de diagnóstico presentaban menos ansiedad estado que los niños ubicados en el resto de los meses $(p<0,05)$. Por último, no resultó estadísticamente significativo el efecto de la variable edad covariada, lo que señala que la variable ansiedad estado no presenta diferencias en los diferentes grupos de edad.

Con respecto a la variable ansiedad rasgo la ANCOVA indicó que no había efectos de interacción entre sexo y tiempo de diagnóstico. De la misma forma, no se encuentran diferencias por sexo, ni tampoco por tiempo de diagnóstico. Al igual que la variable anterior, no resultó significativo el efecto de la variable covariada edad. Esto indica que la variable ansiedad rasgo no presentó diferencias en los diferentes grupos de edad.

En lo concerniente a la variable depresión la ANCOVA señala que no se encuentran efectos de interacción entre sexo y tiempo del diagnóstico. De igual manera, no se encuentran diferencias ni por sexo, ni por tiempo de diagnóstico. Finalmente no resultó significativo el efecto de la variable covariada. Esto indicaría que en los diferentes grupos de edad la variable depresión no presenta diferencias.

\section{DISCUSIÓN}

El objetivo de este estudio fue evaluar los estados emocionales de ansiedad y depresión en niños diagnosticados de cáncer en diferentes fases del tratamiento de la enfermedad y evaluar al mismo tiempo si existían diferencias dependiendo de la edad y sexo.

Los resultados muestran en primer lugar que no se encontraron diferencias en los estados emocionales entre niños y niñas. Esto aparece en primer lugar diferente a lo que se podría esperar ya que es conocido que existen diferencias entre sexo y niveles de ansiedad y depresión (Vera-Villarroel y Buela Casal, 2000). Sin embargo, una posible hipótesis explicativa tiene relación con que el padecer una enfermedad crónica con las características de esta enfermedad podría eliminar las posibles diferencias de acuerdo al sexo que se esperarían en sujetos sanos. De hecho, el padecer esta enfermedad en períodos críticos del desarrollo ocasiona que el desarrollo emocional de los niños tenga algunas características distintas a los niños que no padecen una enfermedad crónica, principalmente en lo que respecta a sus experiencias entre pares y en la interacción con los adultos. De esta forma, tanto el proceso de socialización y de modelamiento por parte de los padres podría influir en la forma de experimentar y/o expresar las emociones.

Por otra parte, al realizar los análisis por grupos de edad tampoco se encontraron diferencias entre los diferentes grupos. Se esperaba que en períodos tan críticos de los niños y el tener algunos años más o menos podría haber influido; sin embargo los datos de este estudio no muestran diferencias en cuanto a la edad 
en las reacciones emocionales en niños con cáncer.

Por último, con respecto específicamente a las variables emocionales no se encontraron diferencias entre los diferentes grupos en ansiedad rasgo ni en depresión. Sin embargo, los análisis mostraron diferencias en la variable ansiedad estado dependiendo del tiempo de que se padecía la enfermedad (esto controlando las variables sexo y edad). De esta forma, los datos mostraron que los niños que padecían por más tiempo la enfermedad manifestaban menos ansiedad estado que los niños con menos tiempo con la enfermedad. Estos resultados coinciden con los planteamientos y estudios que indican que el diagnóstico de una enfermedad y las implicancias que ésta tiene, se convierten en un suceso estresante en la medida que es una situación desconocida e incontrolable para las personas que afecta no sólo su bienestar físico, sino también psicológico (Frank, 1988; Rodríguez y Zurriaga, 1997; Vera-Villarroel y Buela-Casal, 2000). Esto se ve aumentado cuando se trata del diagnóstico de una enfermedad crónica, como el cáncer ya que existe la idea de que esta enfermedad genera consecuencias aversivas para la persona, a lo que se suma su asociación a un pronóstico desfavorable (Bayés, 1985; Sandin, Chorot, Lostao, Valiente y Santed, 1998; Martín et al., 2003). Sin embargo, los datos de nuestro estudio permiten plantear que quizás los niños que llevan más tiempo con la enfermedad podrían estar más habituados a las características de la enfermedad así como también a las exigencias del tratamiento. Posiblemente muchas de las características de la enfermedad estresante como lo incontrolable e impredecible no estén presentes o se presenten en menor medida que los niños que llevan menos tiempo con la enfermedad, de esta forma estos niños presentan precisamente menos ansiedad estado. Esto se relaciona en mayor medi- da si se piensa que justamente la ansiedad estado, es decir de la ansiedad del momento de la evaluación se realizó en el hospital previamente a uno de los controles médicos. Sin embargo el no encontrar ningún tipo de relación o diferencias en las variables de ansiedad rasgo y depresión puede indicar que las variables emocionales más estables no se ven modificadas o alteradas. Lamentablemente no se evaluaron los niveles de depresión estado para dilucidar si, efectivamente la depresión varía o por otra parte sólo se presenta esta tendencia cuando se evalúan aspectos más estables de la depresión y no así en estados más transitorios (Spielberger, Carretero-Dios, De los Santos-Roig, Buela-Casal, 2002a; Spielberger, Carretero-Dios, De los Santos-Roig, Buela-Casal, 2002b, Martín, Grau y Grau, 2003). En este sentido, los datos del presente estudio pueden aportar evidencia de que el mayor tiempo con la enfermedad ha implicado que los niños se adapten al tratamiento, o al menos, a la situación de tratamiento de mejor forma. Futuros estudios que incorporen el control de una mayor cantidad de variables intervinientes así como la posibilidad de estudios longitudinales podrán ayudar a confirmar los resultados del presente estudio.

\section{REFERENCIAS}

Alexander, T., Roodin, P. y Gorman, B. (1994). Psicología evolutiva. Madrid: Pirámide

Aravena, R., Riedemann, A. y Vásquez, A. (1993). La muerte desde el mundo de los niños: una aproximación al concepto de muerte en la infancia. Tesis para optar al título de Licenciado en Psicología. Pontificia Universidad Católica de Chile.

Baider, L. (2003). Cáncer y familia: aspectos teóricos y terapéuticos. Revista Internacional de Psicología Clínica y de la Salud/ International Journal of Clinical and Health Psychology, 3, 505-520. 
Barra, E. (2003). Influencia del estado emocional en la salud física. Terapia Psicológi$\mathrm{ca}, 21,55-60$.

Bayés, R. (1985). Psicología oncológica. Barcelona: Martínez Roca.

Beneit, J. (1994). La enfermedad crónica. En J. Latorre y J. Beneit, J. (Eds.), La Psicología de la salud: Aportaciones para los profesionales de la salud. Buenos Aires: Lavalle.

Berg, S., Grisell, D., DeLaney, T. y Balis, F. (1991). Principles of treatment of pediatric solid tumors. Journal Pediatrics Clinic of North America, 32, 249-265.

Cáceres, C., y Collado, R. (1994). Estandarización del cuestionario de depresión infantil, versión adaptada de Coggiola y Guillón a la población de ambos sexos de 8 a 14 años del Gran Santiago. Tesis para optar al Título de Licenciado en Psicología. Universidad Diego Portales. Chile

Cambiaso, C. y Villaseca, M. (1992). Adaptación y Estandarización del inventario de ansiedad estado-rasgo para niños de 9 a 12 años (STAIC) de la provincia de Santiago. Tesis para optar al título de Licenciado en Psicología. Universidad Diego Portales.

Cruzado y Olivares, J. (1996). Intervención psicológica en pacientes con cáncer. En J.M., Buceta y A. M. Bueno (Eds.), Tratamiento psicológico de hábitos y enfermedades. Madrid: Pirámide.

Frank, J. (1988). Aspectos psicológicos y comportamentales de la enfermedad y del tratamiento. Revista Latinoamericana de Psicología, 20, 45-54.

Galán, A., Pérez San Gregorio, M.A., y Blanco, A. (2000). Análisis del uso de estrategias de afrontamiento de la enfermedad pulmonar obstructiva crónica (EPOC): implicaciones conceptuales. Revista de Psicopatología y Psicología Clínica, 5, 179190.

Kovacs, M. (1992). Children's Depression Inventory. North Tonawanda, NY: MultyHealth Systems.

Latorre y J. Beneit, J. (1994). La Psicología de la salud: Aportaciones para los profesionales de la Salud. Buenos Aires: Lavalle.

Lizasoaín, O. y Polaino-Lorente, A. (1990). Un ejemplo en el ámbito de la pedagogía hospitalaria: los programas de preparación para la hospitalización infantil. En J. González-Simancas y A. Polaino-Lorente (Eds.), Pedagogía hospitalaria. Madrid: Narcea.

Martín, J., Sánchez, Ma. J. y Sierra, J.(2003). Estilos de afrontamiento y apoyo social: su relación con el estado emocional en pacientes de cáncer de pulmón. Terapia Psicológica, 21, 29-37.

Martín, M., Grau, R. y Grau, J (2003). El inventario de depresión estado-rasgo (IDE$\mathrm{RE)}$ : desarrollo de una versión cubana. Terapia Psicológica, 21, 121-135.

Marina, N. (1997). Long-term survivors of childhood cáncer, the medical consequences of cure. Journal Pediatric Clinic of North América, 44, 1021-1042.

Pérez, M.A., Martín, A., Gallego, A., y Santamaría, J.L. (2000). Influencia de algunas variables médicas y psicosociales en la recuperación psicológica de los trasplantados: Futuras líneas de intervención psicológica. Revista de Psicopatología y Psicología Clínica, 5, 71-88.

Pratt, Ch. (1985). Some aspects of childhood cancer epidemiology. Journal Pediatric clinic of North America, 32, 541-556.

Rodríguez, L. y Zurriaga (1997). Estrés, enfermedad y hospitalización. Granada: Escuela Andaluza de Salud Pública.

Rona, E. y Vargas, L. (1994). El impacto psicológico del cáncer en el niño y el adolescente. Revista Chilena de Pediatría, 65, 4855.

Sandin, B., Chorot, P., Valiente, R.M., y Santed, M.A. (1998). Frecuencia e intensidad de los miedos en los niños: Datos normativos. Revista de Psicopatología y Psicología Clínica, 3, 15-25.

Sandin, B., Chorot, P., Lostao, L., Valiente, R.M., y Santed, M.A. (1998). Predictores psicológicos y sociodemográficos de la ansiedad anticipatoria ante la participación en "segundas pruebas» de detección de cáncer de mama. Revista de Psicopatología y Psicología Clínica, 6, 17-36.

Spielberger, C.D. (1973). STAIC Preliminary Manual. Palo Alto, CA: Consulting Psychologist Press.

Spielberger, C.D. (1986). Theory and resarch on anxiety. En C.D. Spielberger (Ed.) 
Anxiety and Behavior. New York: Academics Press.

Spielberger, C.D, Carretero-Dios H, De los Santos-Roig M, Buela-Casal G (2002a). Spanish development version of the state-trait depression questionnaire (ST-DEP): Trait subscale (T-DEP). Revista Internacional de Psicología Clínica y de la Salud /Internacional Journal of Clinical and Health Psy. chology, 2, 51-69.

Spielberger, C.D, Carretero-Dios H, De los Santos-Roig M, Buela-Casal G (2002b). Spanish development version of the statetrait depression questionnaire (ST-DEP): State subscale (S-DEP). Revista Internacional de Psicología Clínica y de la Salud / Internacional Journal of Clinical and Health Psychology, 2, 71-89.

Ulloa, F. (1993). Aspectos psicosociales del cáncer en niños. Revista Chilena de Pediatría, 64, 55-62.
Valverde, V. Y Vasquez, V. (1991). Factores psicosociales en el proceso de ajuste de las familias con un niño con cáncer. Tesis para optar al título de Psicólogo. Pontificia Universidad Católica de Chile.

Vera-Villarroel, P.E., Alvarado, M., Cabrera, P., Urrutia, B. Y Vera, V. (2000). Psychological aspects a cancer diagnosed children group. XXX Congress of the European Association for Behavioural \& Cognitive Therapies. 26-28 Septiembre. Granada.

Vera-Villarroel, P.E. y Buela-Casal, G. (2000). Relaciones entre ansiedad y estilo atributivo. Revista Mexicana de Psicología, 17, 137-142.

Vilches, S., Yañez, R. Y González, M. (1996). Temores del niño a la hospitalización. Revista Horizonte de Enfermería, 7, 3-15.

Wagner, H. y Antic, V.(1997). The problems of pediatric malignancies in the developing world. Journal annals New York Academy of Sciences, 17, 193-204. 